\title{
Timely Access to Genetic Counseling for Women with Epithelial Ovarian Cancer in Nova Scotia
}

\author{
Ashley Warias ${ }^{1}$, Meghan Ferguson² MSc (C)CGC, Erin Chamberlain MSc, CCGC, \\ $\mathrm{BScN}^{3}$, Lauren M. Currie MSc (C)CGC², Nicole Snow MSc, CCGC ${ }^{3}$, Kara Matheson \\ MSc, Lynette S. Penney MD, Katharina Kieser MD, FRCSC, MSc ${ }^{1}$
}

Dalhousie Division of Gynecologic Oncology, Halifax, NS1; Medical Genetics, IWK Health Centre, Halifax, NS (now at MyGeneTeam, Miami, FL)2; Medical Genetics, IWK Health Centre, Halifax, NS ${ }^{3}$, Research Methods Unit, Nova Scotia Health Authority, Halifax, NS ${ }^{4}$, Department of Pediatrics, Faculty of Medicine, Dalhousie University, Halifax, NS

Introduction: Prompt access to genetic counseling and testing is increasingly important for women with epithelial ovarian cancer (EOC) and their families both for risk reduction and targeted therapies. ${ }^{1}$ For many centers the current literature suggests referral rates for testing as low as $19-31 \%{ }^{2,3}$ We evaluated the efficacy of a new collaborative model of care and preferences on timing for genetic counseling in Nova Scotia between Divisions of Medical Genetics and Gynecologic Oncology at Dalhousie University.

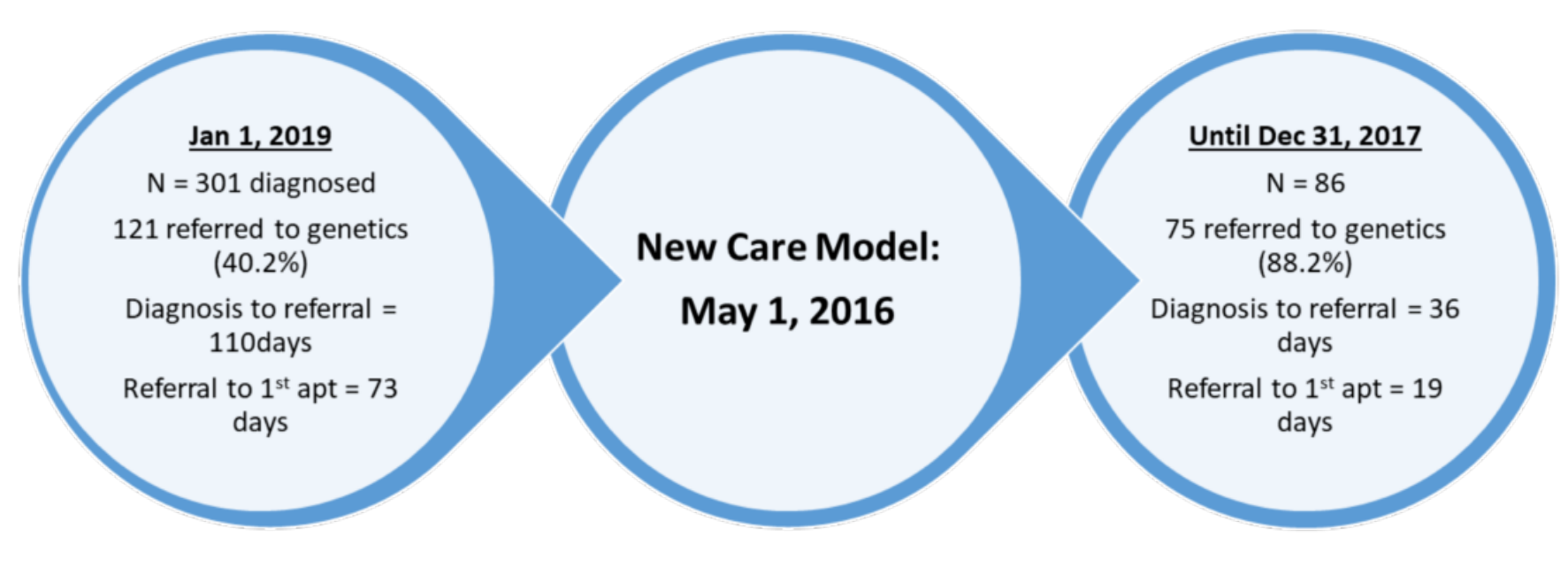

Figure 1: Comparison of Referrals Pre- and Post- New Care Model

Method(s): A retrospective cohort of all women diagnosed with EOC from 2012 to 2017 was identified after ethics approval (NSHA REB \#1022210). Two cohorts were identified, pre and post implementation of a genetic counsellor attending weekly tumor board rounds. Wait-times to different nodes of the clinical pathway assessed. A questionnaire was sent to identify preference for timing of genetic counselling, and factors that may contribute to the decision to pursue genetic testing ( $n=103$ living). Chi-square and Wilcoxon rank sum tests were used to compare demographic and clinical variables pre and post implementation of new model.

Results: There were 387 women with EOC and 196 women were referred for genetic counseling with $89.8 \%$ seen. Most women chose testing after counseling (93.1\%(164/176)). Following the implementation of a new care model in May 2016, there was a $48.2 \%(95 \% \mathrm{Cl} 39.4$ to 56.7$)$ increase in referrals for genetic counseling from $40 \%(121 / 301)$ to $88 \%(75 / 85)$. The wait time from the referral to the first genetic counseling appointment decreased by 67.8 days, $p<0.001$. Survey responses received from $52.6 \%$. Seventy percent of survey respondents wished to be referred for genetic counseling at the time of diagnosis or with initiation of chemotherapy. Women seen for genetic counseling, expressed that the most important reason for pursing genetic testing was for information for their family $(62 \%(36 / 74))$.

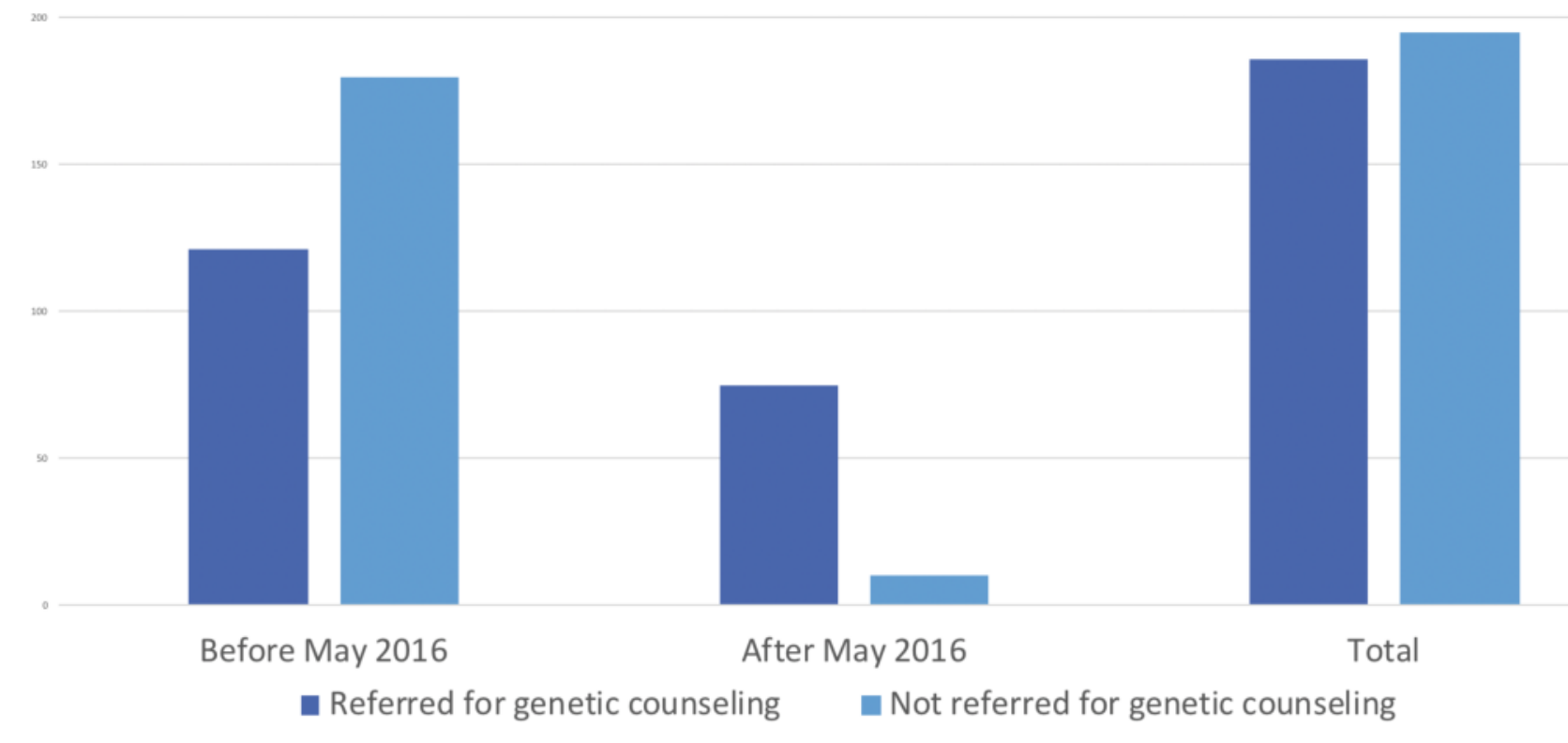

Conclusion: This novel model of care for EOC in Nova Scotia was successful in increasing referral rates for genetic counseling, and genetic testing. Women favored referral to genetic services at the time of diagnosis, highlighting the importance for timely access.

\section{References:}

1. Moore, K. et al (2018). Maintenance Olaparib in Patients with Newly Diagnosed Advanced Ovarian Cancer. New England Journal of Medicine, 379(26), 2495-2505.

2. Demsky, R. et al (2013). Keeping it simple: Genetics referrals for all invasive serous ovarian cancers. Gynecologic Oncology, 130(2), 329-333. http://doi.org/10.1016/j.ygyno.2013.05.003

3. Petzel, S. et al (2013). Genetic risk assessment for women with epithelial ovarian cancer: referral patterns and outcomes in a university gynecologic oncology clinic. Journal of Genetic Counseling, 22(5), 662-673. http://doi.org/10.1007/s10897-013-9598-y

Disclosures: This project was funded by the Atlee Foundation and Astrazeneca 